\title{
Rapid advice guidelines for management of children with COVID-19
}

\author{
Enmei Liu ${ }^{1,2,3 \#}$, Rosalind L. Smyth ${ }^{4,5 \#}$, Zhengxiu Luo ${ }^{1,2,3}$, Amir Qaseem ${ }^{6}$, Joseph L. Mathew ${ }^{7}$ \\ Quan $\mathrm{Lu}^{8}$, Zhou Fu ${ }^{1,2,3}$, Xiaodong Zhao, ${ }^{1,2,3}$, Shunying Zhao ${ }^{9}$, Janne Estill ${ }^{10,11}$, Edwin Shih-Yen Chan ${ }^{12,13}$, \\ Lei Liu ${ }^{14,15}$, Yuan Qian ${ }^{16}$, Hongmei Xu ${ }^{1,2,3}$, Qi Wang ${ }^{17,18}$, Toshio Fukuoka ${ }^{19,20}$, Xiaoping Luo ${ }^{21}$, \\ Gary Wing-Kin Wong ${ }^{22}$, Junqiang Lei $^{23}$, Detty Nurdiati ${ }^{24}$, Wenwei Tu ${ }^{25}$, Xiaobo Zhang ${ }^{26}$, \\ Xianlan Zheng ${ }^{1,2,3}$, Hyeong Sik Ahn ${ }^{27,28,29,30}$, Mengshu Wang ${ }^{23}$, Xiaoyan Dong ${ }^{31}$, Liqun Wu ${ }^{32}$, \\ Myeong Soo Lee ${ }^{33,34,35,36}$, Guobao Li $^{14,15}$, Shu Yang ${ }^{37,38}$, Xixi Feng ${ }^{39}$, Ruiqiu Zhao ${ }^{1,2,3}$, Xiaoxia Lu ${ }^{40}$, \\ Zhihui $\mathrm{He}^{41}$, Shihui Liu ${ }^{42}$, Weiguo Li $^{1,2,3}$, Qi Zhou ${ }^{43}$, Luo Ren ${ }^{1,2,3}$, Yaolong Chen ${ }^{44,45,46,47,48,49}$, Qiu Li ${ }^{1,2,3}$
}

${ }^{1}$ National Clinical Research Center for Child Health and Disorders, Ministry of Education Key Laboratory of Child Development and Disorders, China International Science and Technology Cooperation Base of Child Development and Critical Disorders, Children's Hospital of Chongqing Medical University, Chongqing 400014, China; ${ }^{2}$ Children's Hospital of Chongqing Medical University, Chongqing 400014, China; ${ }^{3}$ Chongqing Key Laboratory of Pediatrics, Chongqing 400014, China; ${ }^{4}$ UCL Great Ormond St Institute of Child Health, London, UK; ${ }^{5}$ Great Ormond Street Hospital, London, UK; ${ }^{6}$ Clinical Policy and Center for Evidence Reviews, American College of Physicians, Philadelphia, USA; ${ }^{7}$ Advanced Pediatrics Centre, PGIMER Chandigarh, Chandigarh, India; ${ }^{8}$ Shanghai Children's Hospital affiliated to Shanghai Jiaotong University, Shanghai 200240, China; ${ }^{9}$ Beijing Children's Hospital, Beijing 100045, China; ${ }^{10}$ Institute of Global Health, University of Geneva, Geneva, Switzerland; ${ }^{11}$ Institute of Mathematical Statistics and Actuarial Science, University of Bern, Bern, Switzerland; ${ }^{12}$ Centre for Quantitative Medicine, Office of Clinical Sciences, Duke-National University of Singapore Medical School, Singapore; ${ }^{13}$ Singapore Clinical Research Institute, Singapore; ${ }^{14}$ National Clinical Research Center for Infectious Disease, Shenzhen 518020 , China; ${ }^{15}$ Shenzhen Third People's Hospital, Shenzhen 518112, China; ${ }^{16}$ Laboratory of Virology, Beijing Key Laboratory of Etiology of Viral Diseases in Children, Capital Institute of Pediatrics, Beijing 100020, China; ${ }^{17}$ Department of Health Research Methods, Evidence and Impact, Faculty of Health Sciences, McMaster University, Hamilton, Canada; ${ }^{18}$ McMaster Health Forum, McMaster University, Hamilton, Canada; ${ }^{19}$ Emergency and Critical Care Center, the Department of General Medicine, Department of Research and Medical Education, Kurashiki Central Hospital, Okayama, Japan; ${ }^{20}$ Advisory Committee in Cochrane Japan, Tokyo, Japan; ${ }^{21}$ Department of Pediatrics, Tongji Hospital, Tongji Medical College, Huazhong University of Science and Technology, Wuhan 430030, China; ${ }^{22}$ Department of Pediatrics, The Chinese University of Hong Kong, Hong Kong, China; ${ }^{23}$ Department of Radiology, the First Hospital of Lanzhou University, Lanzhou 730000, China; ${ }^{24}$ Clinical Epidemiology \& Biostatistics Unit, Department of Obstetrics \& Gynaecology, Faculty of Medicine, Public Health and Nursing, Universitas Gadjah Mada, Yogyakarta, Indonesia; ${ }^{25}$ Department of Pediatrics \& Adolescent Medicine, Li Ka Shing Faculty of Medicine, University of Hong Kong, Hong Kong, China; ${ }^{26}$ Children's Hospital of Fudan University, Shanghai 201102, China; ${ }^{27}$ Department of Preventive Medicine, Korea University, Seoul, Korea; ${ }^{28}$ Korea Cochrane Centre, Seoul, Korea; ${ }^{29}$ Evidence Based Medicine, Seoul, Korea; ${ }^{30}$ Korea University School of Medicine, Seoul, Korea; ${ }^{31}$ Shanghai Children's Hospital, Shanghai 200040, China; ${ }^{32}$ Shenzhen Health Development Research Center, Shenzhen 518028, China; ${ }^{33}$ Korea Institute of Oriental Medicine, Daejeon, Korea; ${ }^{34}$ University of Science and Technology, Daejeon, Korea; ${ }^{35}$ London Southbank University, London, UK; ${ }^{36}$ Tianjin University of Traditional Chinese Medicine, Tianjin 300193, China; ${ }^{37}$ College of Medical Information Engineering, ${ }^{38}$ Digital Institute of Medicine, Chengdu University of Traditional Chinese Medicine, Chengdu 611137, China; ${ }^{39}$ Department of Public Health, Chengdu Medical College, Chengdu 610500, China; ${ }^{40}$ Department of Respiratory Medicine, Wuhan Children's Hospital, Tongji Medical College, Huazhong University of Science and Technology, Wuhan 430015, China; ${ }^{41}$ Chongqing Ninth People's Hospital, Chongqing 400700, China; ${ }^{42}$ Beijing Jishuitan Hospital, Beijing 100035, China; ${ }^{43}$ The First School of Clinical Medicine, Lanzhou University, Lanzhou 730000 , China; ${ }^{44}$ Evidence-based Medicine Center, School of Basic Medical Sciences, Lanzhou University, Lanzhou 730000, China; ${ }^{45}$ WHO Collaborating Centre for Guideline Implementation and Knowledge Translation, Lanzhou 730000, China; ${ }^{46}$ GIN Asia, Lanzhou 730000 , China; ${ }^{47}$ Chinese GRADE Centre, Lanzhou 730000 , China; ${ }^{48}$ Lanzhou University, an Affiliate of the Cochrane China Network, Lanzhou 730000, China; ${ }^{49}$ Key Laboratory of Evidence Based Medicine \& Knowledge Translation of Gansu Province, Lanzhou 730000, China

"These authors contributed equally to this work.

Correspondence to: Yaolong Chen. Evidence-based Medicine Center, School of Basic Medical Sciences, Lanzhou University, Lanzhou 730000, China. Email: chenyaolong@lzu.edu.cn; Qiu Li. the National Clinical Research Center for Child Health and Disorders, Children’s Hospital of Chongqing Medical University, Chongqing 400014, China. Email: liqiu_21@126.com.

Submitted May 06, 2020. Accepted for publication May 22, 2020.

doi: $10.21037 /$ atm-20-3754

View this article at: http://dx.doi.org/10.21037/atm-20-3754 


\section{Introduction}

In December 2019, an infectious disease, caused by a novel coronavirus, emerged in Wuhan City, China. The disease was later named coronavirus disease 2019 (COVID-19) and the virus causing it was named the severe acute respiratory syndrome coronavirus 2 (SARS-CoV-2). The rapid spread of COVID-19 worldwide has brought great challenges to local and global public health security and health systems. On March 12, 2020, the World Health Organization (WHO) declared the coronavirus outbreak a global pandemic and raised the risk of a global SARS-CoV-2 outbreak to "very high" (1-6).

COVID-19, and its pathogen SARS-CoV-2, represent a novel infectious disease and all populations are therefore susceptible to infection. Its basic reproductive number $\mathrm{R}_{0}$ has been estimated at 3.3 (range 1.4 to 6.5 ), which is similar to SARS and much higher than Middle East respiratory syndrome (MERS) or influenza (7-10). By April 15, around two million confirmed cases had been reported over 200 countries worldwide. The exact number of patients under the age of 18 remains unknown, but their percentage among all cases is estimated to be less than $2 \%(11,12)$. Evidence indicates that the family cluster is the main source of COVID-19 infection for children (13). In contrast to adults, most infected children are asymptomatic or have only mild clinical manifestations.

The existing COVID-19 clinical practice guidelines for public health policies have mostly focused on the prevention, diagnosis and treatment in adults, with little attention paid to children. Few of them are based on evidence from systematic reviews (14). Based on the above considerations, an international multidisciplinary working group developed this rapid advice guideline for management of children with COVID-19 using the methods and process proposed by the WHO and GRADE working group (15-17). We present the following article in accordance with the RIGHT reporting checklist (available at http://dx.doi. org/10.21037/atm-20-3754).

\section{Scope and definitions}

This guideline focuses on the management of children younger than 18 years old infected with SARS-CoV-2 (18), including screening, diagnosis, treatment, and patient education. The target users of the guideline include paediatricians, clinical pharmacists, general practitioners, nurses, policy makers, national ministries of health, child rights advocacy groups and other health workers in general and children's hospitals, primary clinics and communities worldwide, as well as families involved in the prevention and control of COVID-19 in children. The definitions for about the terminology and epidemic features of COVID-19 are presented in supplementary.

\section{Guideline working group}

The guideline was launched on January 26, 2020. The 67 members of the working group came from 11 countries and were divided into three groups: (I) a Guideline Development Group (GDG), which comprised 39 panelists from various disciplines, including paediatricians, infectious disease physicians, pulmonologists, epidemiologists, clinical pharmacists, methodologists, nurse practitioners, health economists, general practitioners, legal experts and global health researchers; (II) a Rapid Review Group (RRG), which comprised 26 team members who had experience with conducting systematic reviews; and (III) patient representatives (PR), two guardians of children who were primarily involved in the voting process for recommendations and feedback on the full text of this guideline. All participants were asked to complete a declaration of conflict of interest form.

\section{Guideline development process}

\section{Registration of the guideline and protocol}

The guideline has been registered at the International Practice Guidelines Registry Platform (registration No. IPGRP-2020CN008), and the protocol of the guideline has been published (19). This guideline was developed in accordance with the WHO handbook for guideline development, and the drafting and reporting of the full text followed the RIGHT (Reporting Items for Practice Guidelines in Healthcare) statement $(20,21)$.

\section{Collection and prioritization of clinical questions}

The core members of the GDG including three respiratory paediatricians (EL, RLS, ZL), and a methodologist (YC) initially identified 20 clinical questions, which were emailed to the panelists to rate their importance. The top ten clinical questions were selected for the guideline. The collection and prioritization method of the clinical 
Table 1 Grading of quality of evidence and strength of recommendations

\begin{tabular}{|c|c|}
\hline Quality of evidence & Description \\
\hline Moderate quality of evidence & $\begin{array}{l}\text { We are moderately confident in the effect estimate: the true effect is likely to be close to the estimate of the } \\
\text { effect, but there is a possibility that it is substantially different }\end{array}$ \\
\hline Low quality of evidence & $\begin{array}{l}\text { Our confidence in the effect estimate is limited: the true effect maybe substantially different from the } \\
\text { estimate of the effect }\end{array}$ \\
\hline Strength of recommendation & Description \\
\hline Strong & $\begin{array}{l}\text { Advantages of intervention significantly outweigh disadvantages or disadvantages of intervention } \\
\text { significantly outweigh advantages }\end{array}$ \\
\hline
\end{tabular}

questions is detailed in the protocol.

\section{Evidence syntheses and assessment}

The RRG developed rapid reviews as evidence support for each recommendation. Given the limited number of published studies on COVID-19, for some clinical questions, the RRG also included studies on SARS, MERS and influenza as indirect evidence. GRADE (Grading of Recommendations Assessment, Development and Evaluation) approach was used to assess and rate the quality of evidence and strength of recommendations (Table 1) (16,22). The RRG also drafted the evidence-to-decision (EtD) tables for Delphi surveys.

\section{Formulation of recommendations}

The GDG and PR participated in two rounds of Delphi surveys on February 24 and 28, 2020, discussed the EtD tables, and voted for the preliminary recommendations. A total of 186 suggestions were collected from the panelists. After considering the patients' preferences and values, and the costs, benefits and harms of the interventions, ten recommendations were finally formed. Panelists also reached a consensus on a pathway of management (Figure 1). We will update the recommendations, based on the 10 clinical questions identified in this guideline, based on new evidence in real time as a living guideline on the official websites of The National Clinical Research Center for Child Health and Disorders (https://www.chcmu.com/) and
International Practice Guidelines Registry Platform (http:// www.guidelines-registry.org/).

\section{Recommendations}

Clinical question 1: what are the symptoms of children with COVID-19 and who needs further assessment?

Recommendation 1: the most common symptoms of COVID-19 in children are fever and/or cough, with vomiting and diarrhea occurring less frequently. Children who had contact with COVID-19 patients should be monitored by their guardians for symptoms (weak recommendation, moderate quality of evidence)

\section{Rationale}

Being aware of the main symptoms of COVID-19 in children helps guardians and clinicians to detect suspected cases promptly. Children infected with SARS-CoV-2 are more likely to have only mild symptoms compared with adults, and about one-fifth of children are asymptomatic. Less than half of children present with fever or cough, and one third have both fever and cough. Only a small minority of cases have vomiting and diarrhea.

\section{Evidence summary}

A rapid review included 49 studies (25 case reports, 23 case series and one cohort study) with 1,667 children. The results showed that $83 \%$ [95\% confidence interval (CI): $78 \%$ to $88 \%$ ] of the cases appeared in family clusters, and $48 \%$ (95\% CI: $39 \%$ to $56 \%$ ) of the children had fever, $39 \%$ 


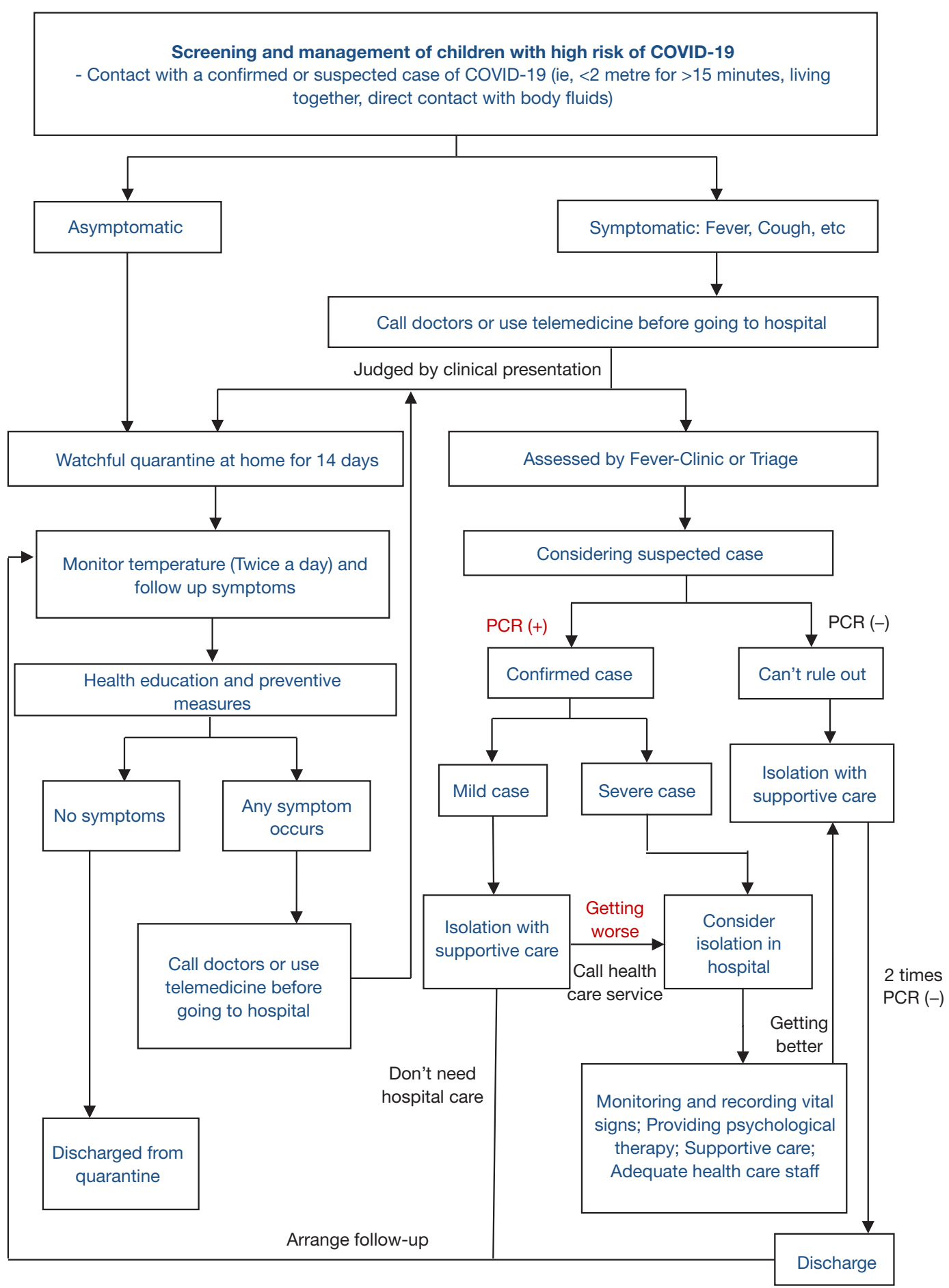

Figure 1 Screening and management of children with high risk of COVID-19. 
(95\% CI: $30 \%$ to $48 \%$ ) cough, $30 \%$ (95\% CI: $18 \%$ to $42 \%$ ) both fever and cough, and $19 \%$ (95\% CI: $14 \%$ to $23 \%$ ) were asymptomatic. Only $3 \%$ (95\% CI: $2 \%$ to $4 \%$ ) of the children had severe symptoms. Seven percent (95\% CI: 5\% to $9 \%$ ) of the children had diarrhea and 6\% (95\% CI: $4 \%$ to $9 \%$ ) of the children had nausea or vomiting (23).

\section{Clinical question 2: how should children who have had contact with COVID-19 patients be managed?}

Recommendation 2: children who have had contact with COVID-19 patients should stay at home for observation for a duration of $\mathbf{1 4}$ days if no symptoms occur. During this time schoolwork should continue and attention should be given to the psychological wellbeing of the children. If there are any symptoms, the guardians should first consult the healthcare system and/ or family doctors (depending on the country-specific arrangements) by telephone call or over the internet (weak recommendation, low quality of evidence) Rationale

Respiratory droplets and contact are the main routes of transmission of SARS-CoV-2. The risk of nosocomial infection of SARS-CoV-2 is high (24). The prognosis of COVID-19 is favorable for children, and deaths have seldom been reported. Guardians should tell children what is happening and why, explaining how long it will continue, providing meaningful activities for them while in quarantine (25). Through telephone or online consultation, family doctors or clinicians can advise about further investigations, depending on the child's clinical condition. A case series suggested that the incubation for children is probably longer than adults (14).

\section{Evidence summary}

According to a review, the basic reproductive number $\mathrm{R}_{0}$ of COVID-19 (range 1.4 to 6.5 ) is roughly similar to that of SARS (range 1.5 to 5), and higher than that of MERS (range 0.3 to 0.8 ) and influenza (range 2 to 3$)(9,10,26,27)$. Most children with SARS-CoV-2 infection have so far been either asymptomatic or presented with only mild symptoms (23). In adults with COVID-19, the incubation period is usually 2 to 14 days (28-31). A rapid review of nosocomial coronavirus infection included 40 studies (case reports and case series) with 22,519 patients, but none of the studies provided direct evidence from children with COVID-19. Among the confirmed patients, the proportions of nosocomial infections with early outbreaks of COVID-19, SARS and MERS were $44 \%$ (95\% CI: $36 \%$ to $51 \%$ ), $36 \%$ (95\% CI: $23 \%$ to $49 \%$ ),
$56 \%$ (95\% CI: $8 \%$ to $100 \%$ ), respectively (32). A rapid review of modelling studies showed that they predicted that isolating people exposed to confirmed or suspected cases would reduce incident cases by between $44 \%$ to $81 \%$ and deaths by between $31 \%$ to $63 \%$, compared with no such measures (33). A rapid review of the application of telemedicine during coronavirus epidemics included nine cross-sectional studies with 100,659 consultations. People were most concerned about symptoms (64.2\%), epidemic situation and public health measures (14.5\%), and psychological problems (10.3\%) during the COVID-19 epidemic. During the SARS epidemic, the proportions of people asking for consultation for symptoms, prevention and $35 \%, 22 \%$ and $23 \%$, respectively. Telemedicine can be helpful in screening suspected patients and providing advice, but the limited possibilities for following up people calling hotlines and difficulties in identifying all suspect cases should be taken into consideration (34).

Clinical question 3: should computed tomography (CT) scan be used for the diagnosis and monitoring of children with COVID-19?

Recommendation 3: CT scan should not be used routinely in the diagnosis of COVID-19 in children, although it may be helpful in monitoring children who develop severe respiratory symptoms (strong recommendation, low quality of evidence)

\section{Rationale}

Radiological examination is widely utilized in the diagnosis of COVID-19 in adults and is recommended by COVID-19 guidelines (35-37). Studies have shown that the symptoms of COVID-19 in children are usually mild, and not associated with any specific changes on a CT scan. While the benefits of a CT scan as a diagnostic tool in children with COVID-19 are uncertain, there is high quality evidence that CT has harmful effects on children (38). After balancing the advantages and disadvantages of CT scan, we suggest not using it routinely in children suspected of having COVID-19. If more evidence on the role of CT for children with severe symptoms emerges, CT scan may be useful for monitoring the severity of the disease.

\section{Evidence summary}

A rapid review included 103 studies (82 case series and 21 case reports) with 5,673 cases. All studies used CT scan as a diagnostic tool for patients with COVID-19. Seven of the included studies focused on children with COVID-19. Using reverse transcription polymerase chain reaction (RT-PCR) 
results as reference, the meta-analysis showed that the pooled sensitivity of chest CT scan in all patients regardless of age was $99 \%$ (95\% CI: $97 \%$ to $100 \%$ ). However, in children the sensitivity of CT was only $66 \%$ (95\% CI: $15 \%$ to $100 \%$ ). The most common imaging manifestation was ground-glass opacities (GGO) which was found in 75\% (95\% CI: 68\% to $82 \%$ ) of the patients. The pooled probability of bilateral involvement was $84 \%$ (95\% CI: $81 \%$ to $88 \%$ ). The role of a chest CT scan in diagnosis of children with COVID-19 is therefore likely to be limited (39).

Clinical question 4: should antiviral drugs such as ribavirin, interferon, remdesivir (GS-5734), lopinavir/ritonavir or oseltamivir be used to treat children with COVID-19?

Recommendation 4: antiviral drugs to treat COVID-19 in children should only be used in the context of clinical trials (strong recommendation, low quality of evidence)

Rationale

Most viral diseases are self-limiting illnesses that do not require specific antiviral therapy. Several guidelines recommend antiviral drugs such as lopinavir/ritonavir (LPV/r), interferon, arbidol and hydroxychloroquine to treat COVID-19 $(36,40,41)$. In China, almost all children with COVID-19 received antiviral therapy (42). However, there is no evidence of the effectiveness of antiviral therapy in children with COVID-19. Published studies have shown that LPV/r and arbidol are not effective against COVID-19, and the efficacy of remdesivir is still controversial $(43,44)$.

\section{Evidence summary}

A rapid review included 23 studies (six randomized controlled trials and 17 cohort studies) with 6,008 patients. None of the studies included direct evidence in children with COVID-19. In adults with COVID-19, the use of $\mathrm{LPV} / \mathrm{r}$ had no effect on mortality [relative risk $(\mathrm{RR})=0.77$, 95\% CI: 0.45 to 1.30 ] and probability of negative a PCR test $(\mathrm{RR}=0.98,95 \% \mathrm{CI}: 0.82$ to 1.18$)$. Arbidol had no benefit on the probability of a negative PCR test $(\mathrm{RR}=1.27$, 95\% CI: 0.93 to 1.73 ). Hydroxychloroquine was effective for promoting the remission of radiographic abnormalities ( $\mathrm{RR}=1.47,95 \% \mathrm{CI}: 1.02$ to 2.11 ) and decreasing the duration of fever [weighted mean difference $(\mathrm{WMD})=-0.90$ days, $95 \%$ CI: -1.48 to -0.31 ], but it was not associated with the probability of a negative PCR result $(\mathrm{RR}=0.93,95 \%$ CI: 0.73 to 1.18 ). There was also no statistically significant difference in the incidence of adverse reactions between the patients receiving the above antiviral drugs and the respective control groups (45).

\section{Clinical question 5: should antibiotics be used to treat children with COVID-19?}

\section{Recommendation 5: antibiotics should not be used for children with COVID-19 if there is no evidence of bacterial coinfection (strong recommendation, moderate quality of evidence) \\ Rationale}

Antibiotics are generally not recommended for viral infections except for patients with concurrent or secondary bacterial infections. Guidelines for COVID-19 consistently recommend against using antibiotics, especially broadspectrum antibiotics. Among the patients with COVID-19, $1.0 \%$ to $27.3 \%$ have secondary bacterial infections, such as Acinetobacter baumannii, Klebsiella pneumoniae, while the proportion of the patients who received antibiotics ranged from $13 \%$ up to $100 \%$. Early prophylactic use of antibiotic agents in SARS patients was likely to cause dysbacteriosis.

\section{Evidence summary}

A rapid review included six studies (five case series, one cohort study) with 626 patients on the efficacy of antibiotics for patients infected with coronavirus, and 33 studies on the present situation of antibiotics use and bacterial coinfections in 3,203 COVID-19 patients. There was no direct evidence from children with COVID-19. In 349 adults with critical MERS, macrolide therapy was not independently associated with a significant difference in 90-day mortality [adjusted odds ratio (OR) $=0.84,95 \%$ CI: 0.47 to 1.51 ] and MERS$\mathrm{CoV}$ RNA clearance [adjusted hazard ratio $(\mathrm{HR})=0.88$, 95\% CI: 0.47 to 1.64] compared with the control group. In 14 SARS patients with secondary infection, antibiotics were effective in relieving symptoms $(50.0 \%)$ and reducing the total number of leukocytes $(61.5 \%)$, supporting the use of antibiotics when there is confirmed bacterial coinfection. Studies on children with COVID-19 indicated that the proportion in which antibiotics were used ranged from $19.4 \%$ to $100 \%$, despite the lack of etiological evidence, with the most common types being meropenem and linezolid. Furthermore, 29 studies on adults with COVID-19 revealed that $13.2 \%$ to $100 \%$ of all patients received antibiotics and the most common types were quinolones, cephalosporins and macrolides. However, only $1.0 \%$ to $27.3 \%$ of the patients had bacterial coinfections; the most common pathogens were Gram-negative bacilli such as Acinetobacter baumannii, Klebsiella pneumoniae and Haemophilus influenzae (46). 
Clinical question 6: should systemic corticosteroids be used to treat children with severe COVID-19?

Recommendation 6: systemic glucocorticoids should not be used routinely for children with COVID-19 (strong recommendation, low quality of evidence). Only low-dose and short-duration systemic glucocorticoid therapy can be used for children with severe COVID-19 in the context of clinical trials (weak recommendation, very low quality of evidence)

Rationale

Systemic glucocorticoids are highly effective anti-inflammatory drugs, but their use in severe respiratory viral infections remains controversial. Evidence has shown that systemic glucocorticoids may have no benefit in severe cases of COVID-19, SARS and MERS, and severe side effects such as femoral head necrosis may occur after high dose administrations.

\section{Evidence summary}

A rapid review included 23 studies (one RCT, 22 cohort studies) with 13,815 patients. There was no direct evidence from children with COVID-19. In adults with COVID-19, the use of systemic glucocorticoids did not reduce mortality ( $\mathrm{RR}=2.00,95 \% \mathrm{CI}: 0.69$ to 5.75 ) or the duration of lung inflammation (WMD $=-1$ day, $95 \% \mathrm{CI}:-2.91$ to 0.91 ). In patients with SARS, glucocorticoids also did not reduce the mortality ( $\mathrm{RR}=1.52,95 \% \mathrm{CI}: 0.89$ to 2.60 ), duration of fever (WMD $=0.82$ days, $95 \%$ CI: -2.88 to 4.52 ) or duration of lung inflammation absorption (WMD $=0.95$ days, $95 \%$ CI: -7.57 to 9.48 ). The use of systemic glucocorticoid therapy prolonged the duration of hospital stay in patients with COVID-19 (WMD =2.43 days, 95\% CI: 1.42 to 3.43), SARS (WMD $=6.83$ days, $95 \%$ CI: 1.48 to 12.17 ) and MERS (WMD $=6.30$ days, $95 \%$ CI: 2.36 to 10.24 ). Longterm use of high-dose glucocorticoids increased the risk of adverse reactions in patients with SARS such as coinfections $(\mathrm{RR}=3.52,95 \%$ CI: 2.33 to 5.32 ) (47).

\section{Clinical question 7: should intravenous immunoglobulin} (IVIG) be used to treat children with severe COVID-19?

Recommendation 7: intravenous immunoglobulin (IVIG) should not be used to treat children with severe COVID-19 (strong recommendation, low quality of evidence)

Rationale

IVIG is an alternative treatment for children with agammaglobulinemia, and an effective treatment for Kawasaki disease. Several Chinese guidelines recommend
IVIG for severe COVID-19 patients (48-50). Onethird of patients with severe COVID-19 have received IVIG in China (51). Unlike convalescent plasma from patients with COVID-19, IVIG does not contain SARSCoV-2 neutralizing antibody (52). Evidence shows that the use of IVIG to treat patients with severe SARS has no significant benefits. Moreover, IVIG may increase the risk of transfusion transmitted diseases and lead to vaccination delay $(53,54)$. In addition, the cost of IVIG is high.

\section{Evidence summary}

A rapid review included six studies (one RCT, four case series and one case report) with 198 patients. There was no direct evidence from children with COVID-19. The survival of adult COVID-19 patients with acute respiratory distress syndrome (ARDS) was not improved by using IVIG $(\mathrm{P}=0.051)$. In adults with severe SARS, IVIG did not reduced mortality $(18.1 \%$ vs. $23.8 \%)$ or the risk of nosocomial coinfections $(65.2 \%$ vs. $52.4 \%)$ compared with the control group, and there was no significant difference in the incidence of nosocomial infection between $(50.0 \% \mathrm{vs}$. $38.5 \%$ ) and ARDS (81.8\% vs. $75.0 \%)$ patients (55).

Clinical question 8: what is appropriate supportive care for children with severe COVID-19?

Recommendation 8: the following forms of supportive care for children with severe COVID-19 are suggested: ensuring sufficient number of adequate health care staff (weak recommendation, low quality of evidence); systematically monitoring and recording vital signs (weak recommendation, low quality of evidence); using supportive care for respiratory and cardiovascular symptoms according to clinical needs (weak recommendation, low quality of evidence); and providing psychological interventions to children and their families when needed (weak recommendation, low quality of evidence)

\section{Rationale}

Supportive treatments are an important and effective part of the management of children with respiratory diseases. Most critically ill patients are treated in the intensive care unit (ICU), and adequate staffing is crucial. The monitoring of vital signs is very important to enable timely intervention in the case of respiratory failure. For critically ill patients, reducing mortality is still the top priority. Therefore, in patients who do not respond adequately to supplemental oxygen therapy, respiratory support should be provided promptly. It is also important to support the circulation and 
fluid balance. Due to the impact of these interventions and the environment in which they are delivered, it is important to provide appropriate learning activities (for example by reading to the child) and psychological support. It is also important to support parents, so healthcare-givers should communicate regularly with them $(56,57)$.

\section{Evidence summary}

An umbrella review included 18 systematic reviews and metaanalyses with COVID-19, SARS, MERS and influenza. The results showed that extracorporeal membrane oxygenation (ECMO) reduced mortality in H1N1 (Influenza A virus subtype H1N1) patients by $25-72 \%$ compared with the control group, but there was no statistically significant difference in the reduction of mortality in patients with ARDS. Besides, the umbrella review showed that increased registered nurse staffing was associated with lower mortality in ICUs (OR $=0.91,95 \%$ CI: 0.86 to 0.96$)$, in surgical patients (OR $=0.84,95 \%$ CI: 0.80 to 0.89$)$, and in medical patients (OR $=0.94,95 \%$ CI: 0.94 to 0.95$)$. Enhanced monitoring of intermittent vital signs was associated with modest reduction in the risk of death when compared with usual care ( $\mathrm{OR}=0.78,95 \% \mathrm{CI}$ : 0.61 to 0.99$)$. In addition, the umbrella review showed that the psychological impact of quarantine is wide-ranging, substantial, and can be long lasting. Officials should quarantine individuals for no longer than required, provide a clear rationale for the quarantine and information about protocols, and ensure sufficient supplies are provided (58).

\section{Clinical question 9: should mothers with COVID-19 continue to breastfeed their babies?}

Recommendation 9: breastfeeding mothers infected with SARS-CoV-2 should continue to breastfeed if their own health permits (strong recommendation, low quality of evidence). Mothers should take appropriate precautions when they come into contact with their babies (strong recommendation, low quality of evidence). If the mother is quarantined without rooming in, her baby may be fed using expressed milk (strong recommendation, low quality of evidence) Rationale

Breast milk is the best source of nutrition for infants, and numerous studies have shown that breastfeeding has multiple benefits. Breastfeeding is recommended by WHO and other international authorities (59). Some guidelines recommend mothers infected with SARS-CoV-2 to suspend breastfeeding while others suggest continuing (60-65). The main transmission routes of SARS-CoV-2 are via droplets and contact. Existing studies have shown that children with COVID-19 tend to have asymptomatic or mild infections. There is no evidence indicating that SARS-CoV-2 can be transmitted via breast milk. We believe that the advantages of breastfeeding outweigh the risks related to the possible infection from the mother. Infected mothers need to take appropriate precautions, such as handwashing before handling their baby and wearing a face mask while in close contact with their baby, to avoid transmission via other routes while breastfeeding.

\section{Evidence summary}

A rapid review included six studies (five case reports, one case series) with 58 breastfeeding mothers. The data contained thirteen samples of breast milk from mothers with COVID-19. The viral nucleic acid tests were negative. There was no direct evidence indicating that SARS$\mathrm{CoV}-2$ could be transmitted by breast milk. The review also contained data from forty-two influenza infected postpartum mothers taking precautions (hand hygiene and wearing masks) before breastfeeding. During one-month of follow-up, no neonates were infected with influenza (65). A systematic review showed wearing masks and hand hygiene (wearing masks: $\mathrm{OR}=0.32,95 \% \mathrm{CI}: 0.26$ to 0.39 and frequent hand washing: $\mathrm{OR}=0.54,95 \% \mathrm{CI}: 0.44$ to $0.67)$ can reduce the risk of respiratory virus transmission via droplets and contact (66).

\section{Clinical question 10: how should parents be advised to get information on SARS-CoV-2 infection?}

Recommendation 10: parents should obtain information from the official websites of authorities such as the WHO and national Centers of Disease Control and Prevention (CDC), or from other sources endorsed by these authorities, rather than from a general search of the internet or social media (strong recommendation, low quality of evidence)

\section{Rationale}

Outbreaks of COVID-19 and other emerging infectious diseases may be associated with considerable fear in the community, because of their evolving nature and inherent uncertainties. Social media is filled with diverse and conflicting information about the epidemic and health education, but surveys have shown that the data posted by government authorities are more reliable. Official websites of the $\mathrm{WHO}$ and most national CDCs update information on the epidemic and preventive measures promptly. 
Guardians need to educate their children on the importance of evidence-based information on COVID-19 and help them practice preventive measures and hygiene behaviors. The latest public health information on these official websites is an essential resource for planning activities such as family travel or overseas studies.

\section{Evidence summary}

A rapid review included six anonymous network sampling surveys after the outbreak of COVID-19 with 15,869 participants, and 18 surveys during SARS and MERS epidemics with over 20,000 participants. There was no direct evidence in health education for parents after COVID-19 outbreak. Many studies revealed that the public did not know how to face emerging infectious diseases. Some surveys showed that people had good knowledge attitude and practice of COVID-19 prevention measures in China, but it is necessary to continue strengthening the public health education of the general population. One study showed that fear and stigmatization of potential SARS patients emerged early in the outbreak, as global media reported dramatic stories from Asia in print media, television and the Internet. One study revealed that emerging health hazards are overreported in mass media in comparison to more familiar threats to public health. Two studies showed that health information from nonprofit, government and academic websites are more accurate than from privately owned commercial and media websites. Five studies demonstrated that religious travelers lacked knowledge of MERS during the outbreak, and conducting health surveys and health education are needed. Three studies from China showed that after a health education intervention, the awareness of how to prevent SARS or MERS was enhanced, and the health behavior was improved (67).

The screening and management of children with high risk of COVID-19 pathways are shown in Figure 1.

\section{Discussion}

\section{Summary}

This guideline covers COVID-19 in children less than 18 years old, and reflects the differences between children and adults in terms of diagnosis, assessment, and management, as well as the needs of children of different ages. For evaluation and diagnosis of children with COVID-19, we believe home observation is an important and appropriate option since most children infected with SARS-CoV-2 are often asymptomatic and present with milder symptoms than adults. CT scan should not be used routinely as an imaging test for diagnosis. Regarding pharmacotherapy, there is no direct clinical evidence for the effectiveness of antiviral drugs, antimicrobial agents, corticosteroids or IVIG in the treatment of children with COVID-19. Considering the potential side effects, resource availability, and patients' preferences related to these interventions, none of these drugs were recommended, except within the context of a clinical trial. Breastfeeding mothers should continue to breastfeed, if their condition permits, but with appropriate protection. Children should first be screened and their families referred for consultation by telemedicine by telephone or Internet, which can promote the rational use of medical resources and reduce the risk of nosocomial infection. Parents and guardians should obtain reliable health information regularly from official channels, and communicate this to their children.

\section{Dissemination and implementation}

This guideline will be published in multiple languages including English, Chinese, Japanese, Russian, German, French, Italian, Vietnamese, Thai, Spanish, Arabic, Portuguese, Polish, Czech, Romanian, Burmese, Hungarian, Hebrew, Hindi, Turkish and Malay. The participants of the guideline working group will promote the guideline first in their own countries, covering a population of 3.65 billion. This guideline will be promoted through WHO Collaborating Centre for Guideline Implementation and Knowledge Translation and Guidelines International Network. The guideline working group will share all the relevant materials related to the guideline, as well as collect feedback from end-users and update the guidelines in the website of International Practice Guidelines Registry Platform and National Clinical Research Center for Child Health and Disorders. We will also develop an easily understandable online patient and public version guideline or brochure based on this guideline, so that children and their caregivers can better understand the advice on the management of COVID-19 in children. Considering the differences in health policies and systems, resources, feasibility and equity across the countries, we will assist countries and regions to adapt the guidelines into their local context.

\section{Strengths and limitations}

Our guideline has several strengths. First, to our knowledge, this guideline is the first international rapid advice guideline 
for management of children with COVID-19 based on WHO guidance approach. Second, our recommendations are supported by rapid reviews and our guideline is based on a systematic review of existing guidelines on COVID-19 (14). Third, the novel coronavirus emerged first in China and most of the early articles on COVID-19 were published in Chinese medical journals. In addition to English-language literature, we reviewed all relevant studies including original clinical data published in Chinese with the help of the National Clinical Research Center for Children Health and Disorders (Chongqing Children Hospital) and the National Clinical Research Center for Infectious Diseases (Shenzhen Third People's Hospital).

Our recommendations should be considered with following limitations: First, the number of confirmed cases in children is still quite small, hence robust evidence is not available. The evidence is still evolving and things may change. Second, most of the evidence is from adults with COVID-19, or extrapolated from other diseases in adults. The generalizability of results from adults to children regarding the mental, physical and social implications of strict quarantine may be questionable. However, many of our recommendations suggest restricting interventions to avoid overdiagnosis and treatment, and the quality of evidence supporting the harmfulness of those interventions is moderate or even high.

\section{Research gaps}

A report by the WHO, UNICEF (United Nations International Children's Emergency Fund) and The Lancet has recently urged future research to focus on children, particularly on the mental health of children during the period of pandemic prevention and control $(68,69)$. Based on the recommendations and evidence of this guideline, we have identified the following research gaps to guide future research:

* How contagious are children infected with SARSCoV-2 compared with adults (viral shedding, viral load, serum antibody)?

* Under what condition should children who have had contact with COVID-19 patients be tested for SARSCoV-2 PCR?

* What is the effectiveness and safety of antiviral drugs for the treatment of children with COVID-19?

* What is the effectiveness and safety of systemic glucocorticoids (low dose and short duration) for the treatment of children with COVID-19?

* How does quarantine affect the psychological condition of children with COVID-19?

* Should public health officials close schools, and what is the effect of school closure on the children and for disease control?

\section{Acknowledgments}

We thank Dr. Sarah Louise Barber, Dr. Yu-Lung Lau, Prof. Youning Liu, and Prof. Jürgen Schwarze for doing external review. We thank Dr. Wilson Were and Mansuk Daniel Han for reviewing the key terms, clinical questions and recommendations. We thank Dr. Yao Zhao, Jihong Dai, Jian Luo, Qubei Li, Donghong Peng, Chang Shu and Daiyin Tian for providing human resources. We thank Dr. Feng Xiao for giving advice on the pathway and terminology. We thank the members of the Rapid Review group for their work (Zijun Wang, Yuyi Tang, Meng Lv, Yinmei Yang, Xufei Luo, Liping Huang, Qianling Shi, Jing Liao, Yangqin Xun, Nan Yang, Qinyuan Li, Yelei Gao, Jingyi Zhang, Rui Liu, Shuya Lu, Muna Baskota, Qingxia Shi, Chenglin Wang, Jianjian Wang, Xia Wang, Xingmei Wang, Xiaoqing Wang, Shuangyuan Yang, Siyi Che, Xin Long, Xin Chen, Wei Li, Hui Zhai).

Funding: National Clinical Research Center for Child Health and Disorders (Children's Hospital of Chongqing Medical University, Chongqing, China) (NCRCCHD2020-EP-01); Special Fund for Key Research and Development Projects in Gansu Province in 2020; The fourth batch of "Special Project of Science and Technology for Emergency Response to COVID-19" of Chongqing Science and Technology Bureau; Special funding for prevention and control of emergency of COVID-19 from Key Laboratory of Evidence Based Medicine and Knowledge Translation of Gansu Province (GSEBMKT2020YJ01); The Fundamental Research Funds for the Central Universities (lzujbky-2020-sp14); Newton international fellowship from The Academy of Medical Science (NIF004/1012); UK National Institute of Health Research GOSH Biomedical Research Centre.

\section{Footnote}

Provenance and Peer Review: This article was submitted to $A T M$ as a revised version along with the incisive peer review comments after rejection from another esteemed journal. Given the revisions and the wide concern and pressing importance of research relating to COVID-19, the article was managed via the rapid communication pathway and 
underwent internal review.

Reporting Checklist: The authors have completed the RIGHT reporting checklist. Available at http://dx.doi.org/10.21037/ atm-20-3754

Conflicts of Interest: All authors have completed the ICMJE uniform disclosure form (available at http://dx.doi. org/10.21037/atm-20-3754). WMW reports that he is currently working for WHO and the position is responsible officer for pediatric care guidelines. The other authors have no conflicts of interest to declare.

Ethical Statement: The authors are accountable for all aspects of the work in ensuring that questions related to the accuracy or integrity of any part of the work are appropriately investigated and resolved.

Open Access Statement: This is an Open Access article distributed in accordance with the Creative Commons Attribution-NonCommercial-NoDerivs 4.0 International License (CC BY-NC-ND 4.0), which permits the noncommercial replication and distribution of the article with the strict proviso that no changes or edits are made and the original work is properly cited (including links to both the formal publication through the relevant DOI and the license). See: https://creativecommons.org/licenses/by-nc-nd/4.0/.

\section{References}

1. Zhu N, Zhang D, Wang W, et al. A novel coronavirus from patients with pneumonia in China. N Engl J Med 2020;382:727-33.

2. Huang C, Wang Y, Li X, et al. Clinical features of patients infected with 2019 novel coronavirus in Wuhan, China. Lancet 2020;395:497-06.

3. Paules CI, Marston HD, Fauci AS. Coronavirus Infections-More Than Just the Common Cold. JAMA 2020;323:707-8.

4. WHO. Naming the Coronavirus Disease (COVID-19) and the Virus That Causes It. Feb 11, 2020. Available online: https://www.who.int/emergencies/diseases/ novel-coronavirus-2019/technical-guidance/naming-thecoronavirus-disease-(covid-2019)-and-the-virus-thatcauses-it (accessed March 16, 2020).

5. Phelan AL, Katz R, Gostin LO. The Novel Coronavirus Originating in Wuhan, China: Challenges for Global Health Governance. JAMA 2020;323:709-10.
6. WHO. WHO characterizes COVID-19 as a pandemic. March 12, 2020. Available online: https://www.who.int/ emergencies/diseases/novel-coronavirus-2019/events-asthey-happen (accessed March 16, 2020).

7. Liu Y, Gayle AA, Wilder-Smith A, et al. The reproductive number of COVID-19 is higher compared to SARS coronavirus. J Travel Med 2020. doi: 10.1093/jtm/taaa021.

8. Wallinga J, Teunis P. Different epidemic curves for severe acute respiratory syndrome reveal similar impacts of control measures. Am J Epidemiol 2004;160:509-16.

9. Kucharski AJ, Althaus CL. The role of superspreading in Middle East respiratory syndrome coronavirus (MERSCoV) transmission. Euro Surveill 2015;20:14-8.

10. Mills CE, Robins JM, Lipsitch M. Transmissibility of 1918 pandemic influenza. Nature 2004;432:904-6.

11. CDC COVID-19 Response Team. Coronavirus Disease 2019 in Children-United States, February 12-April 2, 2020. MMWR 2020;69:422-6.

12. Wu Z, McGoogan JM. Characteristics of and important lessons from the coronavirus disease 2019 (COVID-19) outbreak in China: summary of a report of 72314 cases from the Chinese Center for Disease Control and Prevention. JAMA 2020;323:1239-42.

13. Cai J, Xu J, Lin D, et al. A Case Series of children with 2019 novel coronavirus infection: clinical and epidemiological features. Clin Infect Dis 2020. doi: 10.1093/cid/ciaa198.

14. Zhao S, Cao J, Qian L, et al. A Quality Evaluation of Guidelines on Five Different Viruses Causing Public Health Emergencies of International Concern. Ann Transl Med 2020;8:500.

15. Schünemann HJ, Hill SR, Kakad M, et al. Transparent development of the WHO rapid advice guidelines. PloS Med 2007;4:e119.

16. Guyatt GH, Oxman AD, Vist GE, et al. GRADE: an emerging consensus on rating quality of evidence and strength of recommendations. BMJ 2008;336:924-6.

17. Yang K, Chen Y, Li Y, et al. can China master the guideline challenge? Health Res Policy Syst 2013;11:1.

18. The United Nations International Children's Emergency Fund (UNICEF). Convention on the Rights of the Child. 1989. Available online: https://digitalcommons.ilr.cornell. edu/cgi/viewcontent.cgi? article $=1007 \&$ context $=$ child

19. Li W, Zhou Q, Tang Y, et al. Protocol for the development of a rapid advice guidelines for management of children with SARS-CoV-2 infection. Ann Palliat Med 2020. doi: 10.21037/apm.2020.02.33.

20. WHO. WHO handbook for guideline development. 2014. Available online: https://apps.who.int/medicinedocs/en/m/ abstract/Js22083en/ (accessed March 16, 2020). 
21. Chen Y, Yang K, Marušić A, et al. A reporting tool for practice guidelines in health care: the RIGHT statement. Ann Intern Med 2017;166:128-32.

22. Norris SL, Meerpohl JJ, Akl EA, et al. The skills and experience of GRADE methodologists can be assessed with a simple tool. J Clin Epidemiol 2016;79:150-8.e1.

23. Wang Z, Zhou Q, Wang C, et al. Clinical characteristics of children with COVID-19: a rapid review and meta-analysis. Ann Transl Med 2020;8:620.

24. Wang D, Hu B, Hu C, et al. Clinical Characteristics of 138 Hospitalized Patients With 2019 Novel Coronavirus-Infected Pneumonia in Wuhan, China. JAMA 2020;323:1061-9.

25. Brooks SK, Webster RK, Smith LE, et al. The psychological impact of quarantine and how to reduce it: rapid review of the evidence. Lancet 2020;395:912-20.

26. Liu Y, Gayle AA, Wilder-Smith A, et al. The reproductive number of COVID-19 is higher compared to SARS coronavirus. J Travel Med 2020. doi: 10.1093/jtm/taaa021.

27. Lloyd-Smith JO, Galvani AP, Getz WM. Curtailing transmission of severe acute respiratory syndrome within a community and its hospital. Proc Biol Sci 2003;270:1979-89.

28. Guan WJ, Ni Z, Hu Y, et al. Clinical characteristics of coronavirus disease 2019 in China. N Engl J Med 2020. doi: 10.1056/NEJMoa2002032.

29. Linton NM, Kobayashi T, Yang Y, et al. Incubation period and other epidemiological characteristics of 2019 novel coronavirus infections with right truncation: a statistical analysis of publicly available case data. J Clin Med 2020;9:E538.

30. Backer JA, Klinkenberg D, Wallinga J. Incubation period of 2019 novel coronavirus (2019-nCoV) infections among travellers from Wuhan, China, 20-28 January 2020. Euro Surveill 2020;25:2000062.

31. Li Q, Guan X, Wu P, et al. Early Transmission Dynamics in Wuhan, China, of Novel Coronavirus-Infected Pneumonia. N Engl J Med 2020;382:1199-207.

32. Zhou Q, Gao Y, Wang X, et al. Nosocomial infections among patients with COVID-19, SARS and MERS: a rapid review and meta-analysis. Ann Transl Med 2020;8:629.

33. Nussbaumer-Streit B, Mayr V, Dobrescu AI, et al. Quarantine alone or in combination with other public health measures to control COVID-19: a rapid review. Cochrane Database Syst Rev 2020;4:CD013574.

34. Gao Y, Liu R, Zhou Q, et al. Application of telemedicine during the coronavirus disease epidemics: a rapid review and meta-analysis. Ann Transl Med 2020;8:626.

35. Jin YH, Cai L, Cheng Z, et al. A rapid advice guideline for the diagnosis and treatment of 2019 novel coronavirus (2019-nCoV) infected pneumonia (standard version). Mil Med Res 2020;7:4.

36. Maternal and Fetal Physician Professional Committee of the Chinese Physician Association of Obstetricians and Gynecologists. Expert advice on new coronavirus infections during pregnancy and puerperium. Chin J Perinatal Med 2020;23:73-9.

37. Chinese Medical Association Radiology Branch. Radiological diagnosis of novel coronavirus pneumonia: expert recommendations from the Chinese Medical Association Radiology Branch. Chin J Radiol 2020. doi: 10.3760/cma.j.issn.1005-1201.2020.0001.

38. Mathews JD, Forsythe AV, Brady Z, et al. Cancer risk in 680,000 people exposed to computed tomography scans in childhood or adolescence: data linkage study of 11 million Australians. BMJ 2013;346:f2360.

39. Lv M, Wang M, Yang N, et al. Chest computed tomography for the diagnosis of patients with coronavirus disease 2019 (COVID-19): a rapid review and meta-analysis. Ann Transl Med 2020;8:622.

40. Shen K, Yang Y, Wang T, et al. Diagnosis, treatment, and prevention of 2019 novel coronavirus infection in children: experts' consensus statement. World J Pediatr 2020. doi: 10.1007/s12519-020-00343-7.

41. Chen ZM, Fu J, Shu Q, et al. Diagnosis and treatment recommendations for pediatric respiratory infection caused by the 2019 novel coronavirus. World J Pediatr 2020. doi: 10.1007/s12519-020-00345-5.

42. Qiu H, Wu J, Hong L, et al. Clinical and epidemiological features of 36 children with coronavirus disease 2019 (COVID-19) in Zhejiang, China: an observational cohort study. Lancet Infect Dis 2020. doi: 10.1016/S1473-3099(20)30198-5.

43. Grein J, Ohmagari N, Shin D, et al. Compassionate use of remdesivir for patients with severe COVID-19. N Engl J Med 2020. doi: 10.1056/NEJMoa2007016.

44. Wang Y, Zhang D, Du G, et al. Remdesivir in adults with severe COVID-19: a randomised, double-blind, placebocontrolled, multicentre trial. Lancet 2020. doi: 10.1016/ S0140-6736(20)31022-9.

45. Shi Q, Zhou Q, Wang X, et al. Potential effectiveness and safety of antiviral agents in children with coronavirus disease 2019: a rapid review and meta-analysis. Ann Transl Med 2020;8:624.

46. Wang J, Tang Y, Ma Y, et al. Efficacy and safety of antibiotic agents in children with COVID-19: a rapid review. Ann Transl Med 2020;8:619.

47. Lu S, Zhou Q, Hang L, et al. Effectiveness and safety of 
glucocorticoids to treat COVID-19: a rapid review and meta-analysis. Ann Transl Med 2020;8:627.

48. Chen Z, Fu J, Shu Q, et al. Diagnosis and treatment recommendation for pediatric coronavirus disease-19. Zhejiang Da Xue Xue Bao Yi Xue Ban 2020;49:1.

49. Pediatric Branch of Guangdong Medical Association. Expert consensus on diagnosis and treatment of new coronavirus pneumonia in paediatrics of guangdong province. Guangdong Med 2020;41:217-21.

50. Chinese society of pediatrics. Recommendations for diagnosis and prevention of 2019 novel coronavirus infection in children (1 edition). Chinese Journal of Pediatrics 2020;58:169-74.

51. Guan W, Ni Z, Hu Y, et al. Clinical characteristics of 2019 novel coronavirus infection in China. medRxiv 2020. doi: 10.1101/2020.02.06.20020974.

52. Kazatchkine MD, Kaveri SV. Immunomodulation of autoimmune and inflammatory diseases with intravenous immune globulin. N Engl J Med 2001;345:747-55.

53. Desborough MJ, Miller J, Thorpe SJ, et al. Intravenous immunoglobulin-induced haemolysis: a case report and review of the literature. Transfus Med 2014;24:219-26.

54. Listed NA. A guide to contraindications to childhood vaccinations. Paediatrics \& Child Health 2000;5:11-2.

55. Zhang J, Yang Y, Yang N, et al. Effectiveness of intravenous immunoglobulin for children with severe COVID-19: a rapid review. Ann Transl Med 2020;8:625.

56. Chan SS, Leung D, Chui H, et al. Parental response to child's isolation during the SARS outbreak. Ambul Pediatr 2007;7:401-4.

57. Chan SS, Leung DY, Wong EM, et al. Balancing infection control practices and family-centred care in a cohort of paediatric suspected severe acute respiratory syndrome patients in Hong Kong. J Paediatr Child Health 2006;42:20-7.

58. Luo X, Lv M, Wang X, et al. Supportive care for patient with respiratory diseases: an umbrella review. Ann Transl Med 2020;8:621.

59. WHO. Guideline: Protecting, Promoting and Supporting. Breast feeding in Facilities Providing Maternity and Newborn Services. 2017. Available online: https://www. who.int/nutrition/publications/guidelines/breastfeedingfacilities-maternity-newborn/en/ (accessed Mar 30 2020).

60. NHC. Department of Maternal and Child Health of National Health Council, Notice on strengthening maternal disease treatment and safe midwifery during the prevention and control of COVID-19. February 8, 2020. Available online: http://www.nhc.gov.cn/fys/s3581/202002/4f80657b3 46e4d6ba76e2cfc3888c630.shtml (accessed Mar 30 2020).

61. NCIRD. Interim Guidance on Breastfeeding for a Mother
Confirmed or Under Investigation For COVID-19. March 17, 2020. Available online: https://www.cdc.gov/ coronavirus/2019-ncov/specific-groups/pregnancyguidance-breastfeeding.html (accessed Mar 30 2020).

62. NCIRD. Division of Viral Diseases, Interim Considerations for Infection Prevention and Control of Coronavirus Disease 2019 (COVID-19) in Inpatient Obstetric Healthcare Settings. February 18, 2020. Available online: https://www.cdc.gov/coronavirus/2019ncov/hcp/inpatient-obstetric-healthcare-guidance.html\#an chor_1582067978854Interim (accessed Mar 30 2020).

63. WHO. Clinical management of severe acute respiratory infection when COVID-19 is suspected. March 12, 2020. Available online: https://www.who.int/publicationsdetail/clinical-management-of-severe-acute-respiratoryinfection-when-novel-coronavirus-(ncov)-infection-issuspected (accessed Mar 30 2020).

64. AAP. Management of Infants Born to Mothers with COVID-19. April 2, 2020. Available online: https:// services.aap.org/en/pages/2019-novel-coronavirus-covid19-infections/faqs-management-of-infants-born-to-covid19-mothers (accessed Mar 30 2020).

65. Yang N, Che S, Zhang J, et al. Breastfeeding of infants born to mothers with COVID-19: a rapid review. Ann Transl Med 2020;8:618.

66. Jefferson T, Del Mar CB, Dooley L, et al. Physical interventions to interrupt or reduce the spread of respiratory viruses. Cochrane Database Syst Rev 2011;7:CD006207.

67. Li W, Liao J, Li Q, et al. Public health education for parents during the outbreak of COVID-19: a rapid review. Ann Transl Med 2020;8:628.

68. Clark H, Coll-Seck AM, Banerjee A, et al. A future for the world's children? A WHO-UNICEF-Lancet Commission. Lancet 2020;395:605-8.

69. Wang G, Zhang Y, Zhao J, et al. Mitigate the effects of home confinement on children during the COVID-19 outbreak. Lancet 2020;395:945-7.

Cite this article as: Liu E, Smyth RL, Luo Z, Qaseem A, Mathew JL, Lu Q, Fu Z, Zhao X, Zhao S, Estill J, Chan ESY, Liu L, Qian Y, Xu H, Wang Q, Fukuoka T, Luo X, Wong GWK, Lei J, Nurdiati D, Tu W, Zhang X, Zheng X, Ahn HS, Wang M, Dong X, Wu L, Lee MS, Li G, Yang S, Feng X, Zhao R, Lu X, He Z, Liu S, Li W, Zhou Q, Ren L, Chen Y, Li Q. Rapid advice guidelines for management of children with COVID-19. Ann Transl Med 2020;8(10):617. doi: $10.21037 /$ atm-20-3754 


\section{Basic definitions}

\section{Asymptomatic infection}

Children who have been exposed to a confirmed or suspected case of COVID-19, and are tested positive for SARS-CoV-2 without manifestations of any clinical symptoms and abnormal chest imaging findings $(40,70)$.

\section{Confirmed case}

Suspected case with at least one of the following types of pathogenic evidence (70):

(I) Positive result for SARS-CoV-2 by reverse transcription-polymerase chain reaction (RT-PCR) test for nucleic acid in respiratory or blood or stool samples.

(II) Viral gene sequencing showing high homogeneity to SARS-CoV-2 in respiratory or blood or stool samples.

\section{Contact}

Contact is defined as a person who is involved in any of the following (70):

(I) Staying in the same close environment of a COVID-19 patient (including apartments, houses, classroom, gatherings).

(II) Traveling together in close proximity $(1 \mathrm{~m})$ with a COVID-19 patient in any kind of transport.

(III) Direct exposure to body fluids or specimens including aerosols.

There are so far no separate criteria for contact specifically for children.

\section{Incubation period}

Incubation period refers to the interval between exposure to SARS-CoV-2 and the symptom onset. Incubation period of COVID-19 is usually 1 to 14 days, and in most cases between 3 and 7 days (71). The longest incubation period observed was 24 days in one study (28).

\section{Mild case}

Children with a SARS-CoV-2 infection who present upper respiratory tract infection (with fever, cough and/or fatigue), without manifestation of pneumonia $(40,72)$.

\section{Route of transmission}

The main route of the transmission of SARS-CoV-2 is through respiratory droplets and contact (73). Transmission may occur through aerosol when exposed to high concentrations of aerosols in a relatively closed environment for a long time (74) and fecal-oral route (75). Whether transmission can occur through urinary tract, or from mother to infant either in utero (76), perinatally or through breast milk, has not been established yet.

\section{Quarantine}

If children are asked to quarantine, they should stay at home and avoid going to a public area or attending social gatherings for 14 days after the date of their suspected epidemic contact or exposure to SARS-CoV-2. In addition, their caregivers should report any symptoms of COVID-19 immediately to their health providers. Appropriate personal protective equipment and hygiene practices should be used when contact is needed $(77,78)$.

Severe acute respiratory syndrome coronavirus 2 (SARS$\mathrm{CoV}-2)$

A novel coronavirus $(\mathrm{CoV})$ is a new strain of coronavirus that has not been previously identified in humans. The virus that caused the outbreak of respiratory illness first detected in Wuhan, China, is named Severe Acute Respiratory Syndrome Coronavirus 2 (SARS-CoV-2) (79). This is an enveloped RNA virus belonging to the genus Betacoronavirus (80), with a diameter of $60-140 \mathrm{~nm}$. The genetic characteristics of SARS-CoV-2 are different from those of the severe acute respiratory syndrome coronavirus (SARS-CoV) and the Middle East respiratory syndrome coronavirus (MERS-CoV) (80). The genome of SARS$\mathrm{CoV}-2$ is suggested to be $88 \%$ identical with the genome of two bat-derived SARS-like coronaviruses (bat-SLCoVZC45 and bat-SL-CoVZXC21) (80). The official name given by the World Health Organization to the disease caused by SARS-CoV-2 is coronavirus disease 2019 (COVID-19) (79).

\section{Severe case}

Children with COVID-19 who fulfill the criteria of severe pneumonia (such as tachypnoea, dyspnea, respiratory distress, hypoxemia, change of consciousness, or trouble 
feeding) or critical illness (such as respiratory failure, septic shock or other organ failure requiring intensive care) $(72,81)$.

\section{Suspected case (person under investigation)}

Children who had a history of contact with COVID-19 patients and have any of the following conditions:

(I) Any acute viral respiratory illness (fever and/or respiratory symptoms) (70);

(II) Other symptoms such as gastrointestinal symptoms (vomiting and/or diarrhea) (28);

(III) Abnormal chest imaging findings with no symptom (82).

\section{References}

70. World Health Organization (WHO). Global Surveillance for human infection with novel coronavirus (2019-nCoV) Interim guidance. 2020. Available online: https://www. who.int/publications-detail/global-surveillance-forhuman-infection-with-novel-coronavirus-(2019-ncov) (accessed Feb 27, 2020).

71. Li Q, Guan X, Wu P, et al. Early Transmission Dynamics in Wuhan, China, of Novel CoronavirusInfected Pneumonia. N Engl J Med 2020. doi: 10.1056/ NEJMoa2001316.

72. World Health Organization (WHO). Clinical management of severe acute respiratory infection when novel coronavirus (nCoV) infection is suspected. 2020. Available online: https://www.who.int/publications-detail/clinicalmanagement-of-severe-acute-respiratory-infection-whennovel-coronavirus-(ncov)-infection-is-suspected (accessed Mar 13, 2020).

73. Centers for Disease Control and Prevention (CDC). How COVID-19 Spreads. 2020. Available online: https://www. cdc.gov/coronavirus/2019-ncov/about/transmission.html (accessed Mar 4, 2020).

74. National Health Commission of the People's Republic of China (NHC). New coronavirus pneumonia prevention and control program (7nd ed.) (in Chinese). 2020. Available online: http://www.nhc.gov.cn/yzygj/s7653p/202 003/46c9294a7dfe4cef80dc7f5912eb1989/files/ce3e69458 32a438eaae415350a8ce964.pdf (accessed Mar 3, 2020).
75. Xiao F, Tang $M$, Zheng $X$, et al. Evidence for gastrointestinal infection of SARS-CoV-2. Gastroenterology 2020. doi: 10.1053/j.gastro.2020.02.055.

76. Chen H, Guo J, Wang C, et al. Clinical characteristics and intrauterine vertical transmission potential of COVID-19 infection in nine pregnant women: a retrospective review of medical records. Lancet 2020;395:809-15.

77. Centers for Disease Control and Prevention (CDC). Interim Guidance for Preventing the Spread of Coronavirus Disease 2019 (COVID-19) in Homes and Residential Communities. Available online: https://www. cdc.gov/coronavirus/2019-ncov/hcp/guidance-preventspread.html (accessed Mar 6, 2020).

78. World Health Organization (WHO). Home care for patients with suspected novel coronavirus (nCoV) infection presenting with mild symptoms and management of contacts. 2020. Available online: https://www.who. int/publications-detail/home-care-for-patients-withsuspected-novel-coronavirus-(ncov)-infection-presentingwith-mild-symptoms-and-management-of-contacts (accessed Feb 04, 2020).

79. World Health Organization (WHO). Naming the coronavirus disease (COVID-2019) and the virus that causes it. 2020. Available online: https://www.who.int/ emergencies/diseases/novel-coronavirus-2019/technicalguidance/naming-the-coronavirus-disease-(covid-2019)and-the-virus-that-causes-it (accessed Mar 15, 2020).

80. Lu R, Zhao X, Li J, et al. Genomic characterisation and epidemiology of 2019 novel coronavirus: implications for virus origins and receptor binding. Lancet 2020;395:565-74.

81. World Health Organization (WHO). Pocket book of hospital care for children: Guidelines for the management of common childhood illnesses. 2013. Available online: https://apps.who.int/iris/bitstream/han dle/10665/81170/9789241548373_eng.pdf;jsessionid=C 5285C6B799D7D1036F9354B896D2C32? sequence=1 (accessed Mar 15, 2020).

82. Chan JF, Yuan S, Kok KH, et al. A familial cluster of pneumonia associated with the 2019 novel coronavirus indicating person-to-person transmission: a study of a family cluster. Lancet 2020;395:514-23. 